\title{
The 10q25.3-26.1 G protein-coupled receptor gene GPR26 is epigenetically silenced in human gliomas
}

\author{
JEAN-LOUIS BOULAY ${ }^{1}$, MIHAI-CONSTANTIN S. IONESCU ${ }^{1}$, BALASUBRAMANIAN SIVASANKARAN ${ }^{1}$, \\ MARTIN LABUHN $^{1}$, BÉATRICE DOLDER-SCHLIENGER ${ }^{1}$, ELISABETH TAYLOR ${ }^{1}$, PIER MORIN Jr ${ }^{2}$, \\ BRIAN A. HEMMINGS ${ }^{2}$, MARIA MADDALENA LINO ${ }^{1}$, GRAHAM JONES $^{1}$, DANIEL MAIER $^{1}$ and ADRIAN MERLO ${ }^{1}$ \\ ${ }^{1}$ Laboratory of Molecular Neuro-Oncology, Department of Biomedicine, University Hospital, \\ CH-4031 Basel; ${ }^{2}$ Friedrich Miescher Institute, CH-4058 Basel, Switzerland
}

Received March 20, 2009; Accepted May 15, 2009

DOI: 10.3892/ijo_00000428

\begin{abstract}
Loss of heterozygosity (LOH) of the entire chromosome 10 is the most frequent genetic alteration in human glioblastoma (GBM). In addition to PTEN/MMAC1 on 10q23.3, clustering of partial deletion break-points on 10q25.326.1 points to a second suppressor locus. The proposed target gene DMBT1 was not confirmed. By somatic deletion mapping of this region, we identified the complementary DNA encoding the human homologue of rat orphan $G$ proteincoupled receptor GPR26. GPR26 is highly expressed in fetal and adult brain, but frequently reduced or absent in glioma cells and biopsies, due to de novo methylation of its $5^{\prime} \mathrm{CpG}$ island. Silencing of GPR26 was reversed with 5-aza-deoxycytidine and the histone deacetylase inhibitor trichostatin A. Furthermore, overexpression of GPR26 in HEK and in U87 glioma cells increased intracellular cAMP concentration which is considered to induce astrocytic differentiation. Interestingly, we observed concomitant silencing of GPR26 with $O^{6}$-methylguanine-DNA methyl transferase (MGMT), a DNA repair gene co-localized on 10q25.3-26.1 ( $\mathrm{p}=0.0001)$. We conclude that epigenetic silencing is a common mechanism in malignant gliomas that simultaneously inactivates $M G M T$ and GPR26. The 10q25.3-26.1 region may contain an important epigenetic pathway in brain tumorigenesis.
\end{abstract}

\section{Introduction}

Loss of heterozygosity ( $\mathrm{LOH}$ ) of the entire chromosome 10 is the most frequent genetic alteration observed in human glioblastoma (GBM). Initial deletion studies on this chromosome led to the identification of the PTEN/MMACl suppressor gene, located on chromosome band 10q23.3. This gene was

Correspondence to: Dr Jean-Louis Boulay, Laboratory of Molecular Neuro-Oncology, Department of Research, University Hospital, CH-4031 Basel, Switzerland

E-mail: jean-louis.boulay@unibas.ch

Key words: malignant glioma, 10q25.3-26.1, epigenetic silencing, G protein-coupled receptor, cyclic AMP not only mutated in glioblastomas, but also in a wide range of human cancers with 10q loss (1-6).

Further clustering of partial chromosome $10 \mathrm{q}$ deletion break-points by somatic deletion mapping in malignant gliomas suggested the existence of a second more telomeric tumor suppressor locus in the region 10q25.3-26.1 (7-11) Although a gene designated Deleted in Malignant Brain Tumor 1 (DMBT1) had been identified at this locus $(12,13)$, its function in tumor suppression of glioma has remained speculative (14).

More distally, the region 10q25.3-26.1 encodes the gene for the DNA repair protein $\mathrm{O}^{6}$-methylguanine-DNA methyl transferase (MGMT). Presence of MGMT prevents carcinogenesis by alkylating agents (15). Allelic variants of the $M G M T$ gene have been associated with gliomagenesis (16). In fact, the $M G M T$ gene promoter is dominantly inactivated by frequent 10q25.3-q26.1 DNA methylation in glioma, and MGMT epigenetic silencing turned out to be relevant for patient survival providing a survival advantage when treated with alkylating agents (17-19). However, there is no evidence for a tumor suppressor pathway in which MGMT may be involved, and its gene is located further telomeric to the defined minimally lost area $(3,9)$.

The minimally lost area commonly deletes the gene encoding the homologue of the rat orphan $\mathrm{G}$ protein-coupled receptor GPR26 (20) that is further inactivated by epigenetic silencing. Restoration of GPR26 expression resulted in increased intracellular cAMP levels, a suppressor of tumor progression. This defines GPR26 as a novel target for 10q25.3-q26.1 epigenetic silencing in glioma.

\section{Materials and methods}

Primary tumors and cell lines. BS series are primary tumor tissues obtained from patients diagnosed with primary CNS tumors, were classified according to the World Health Organization (WHO) grading system. Secondary GBM (GBMII) were distinguished from primary GBM (GBMI) when a record of earlier stage of glioma was available (21). All biopsies showed allelic loss of entire 10q or at least of the 10q25.3-26.1 area (9). Normal brain tissue used as template for microarray was obtained from samples of brain surgery for non-neoplastic disease. Human brain tumor cell lines 
LN18, LN71, LN215, LN235, LN308, LN319, LN340, LN401, LN405, LN427, LN428, U87, U343, derived from adult patients with de novo glioblastoma were selected for this study (22). The LN cell lines were kindly provided by Erwin van Meir and Nicolas de Tribolet, Lausanne, Switzerland. Cell lines U87 and U343 were obtained from the American Type Culture Collection (ATCC, Rockville, MD, USA). All cell lines, showed loss of heterozygosity at 10q25.3-26.1 $(3,9)$ and the genetic status of $p 53, p 16, p 14^{A R F}$, PTEN tumor suppressor genes has been reported $(3,22)$. Cells were cultured using DMEM supplemented with $10 \%$ fetal calf serum and standard antibiotics. Cells were transfected by $\mathrm{CaCl}_{2}$ precipitation. Stably transfected cells were selected by addition of $50 \mu \mathrm{g} / \mathrm{ml}$ Geneticin ${ }^{\circledR}$ into the culture medium for 15-20 days.

Expressed sequence tag markers. Following expressed sequence tags (EST) comprised between 10q25.3-26.1 STS markers D10S221 and D10S575 (Fig. 1) were selected for further analyses: stSG45253, stSG30245, A006D08, WI-17804, WI-16905, Bdy77b02, WI-14638, SGC33199, sts-N35985, SGC35172, WI-16392, WI-17828, WI-15113, WI-18655, stSG29862, stSG3812, WI-6602, stSG1556, A005K22, stSG8848, KIAA0140, stSG13277, SGC38118, SGC30414, A008K16, WI-17472.

RNA extraction and reverse-transcription. Total RNA from biopsies and cell lines was extracted using TRIzol (Life Technologies, Gaithersburg, USA) and reverse-transcribed with Superscript RNaseH-reverse transcriptase according to the manufacturer's instructions (Life Technologies). Human fetal brain RNA and cDNA were purchased from Clontech (Palo Alto, CA, USA).

Genomic and transcript analyses of ESTs. Genomic and complementary DNAs were amplified by PCR using the EST markers described above. Multiplex PCR was performed using GAPDH to control PCR amplification.

Extension of EST WI-6602. The phagemid clone 21465 containing the WI-6602 sequence was obtained from the Image consortium library (UK HGMP Resource Centre, Hinxton, Cambridge, UK) and sequence was completed using the ABI sequencing system (Perkin-Elmer, Foster City, CA, USA). The 5'-end of the corresponding phagemid was extended by rapid amplification cDNA ends (RACE) from human fetal brain cDNA (Clontech), with primers gga gattgaaccgtttggagaattagggaa and ccctccctcagaaaggtgcca. Confirmation that Hs.278719 and extended Hs.1275 are part of the same transcription unit was provided by RT-PCR on whole fetal brain cDNA template with primers gccac caagaagatcagcaccttca and gagacgaggtgccatgttagatc. Open reading frame (ORF) and sequence homologies were searched through the web resources at the US National Center for Biotechnology Information (NCBI): http://www. ncbi.nlm.nih.gov/BLAST and http://www.ncbi.nlm.nih. gov/gorf/gorf.html, respectively. Multiple sequence alignment was performed with the T-Coffee program available on-line at the Swiss EMBnet node server thttp://www.ch.embnet.org/ index.html.

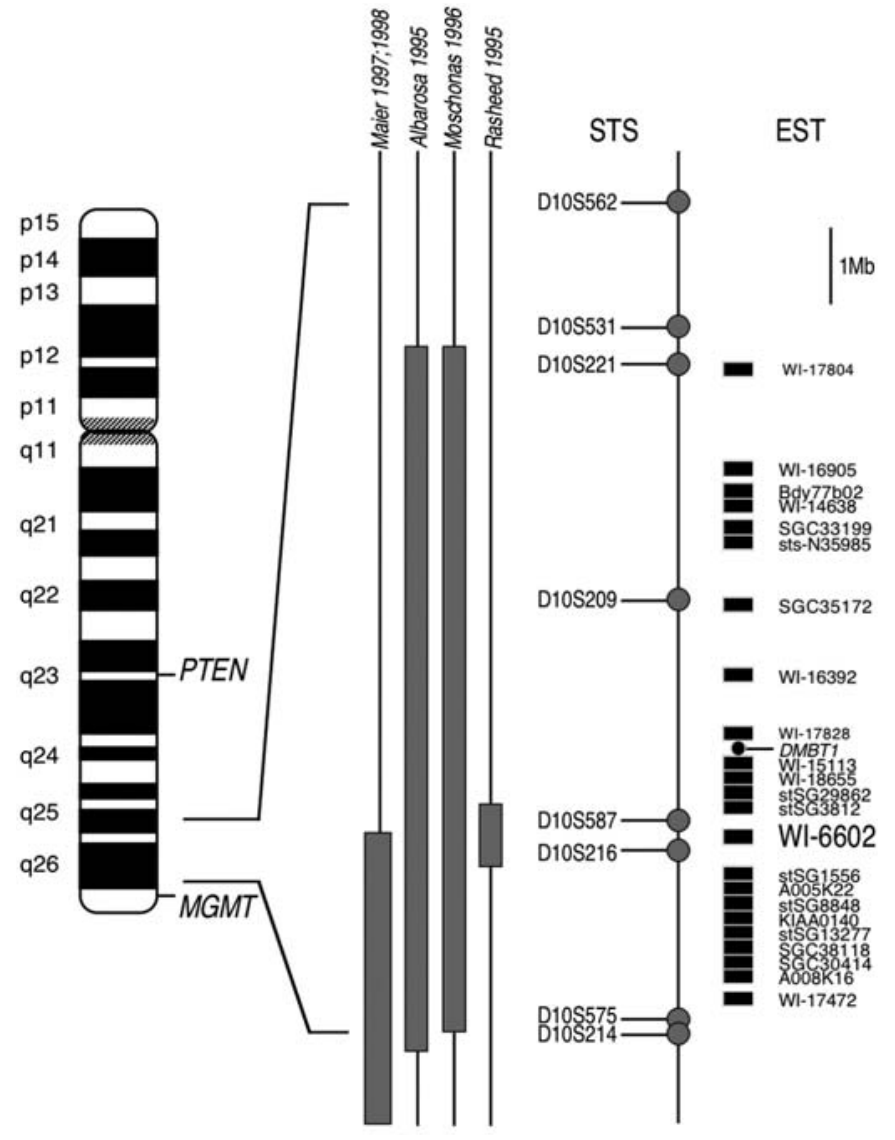

Figure 1. Compilation of somatic deletion mapping studies on 10q25.3-26.1. Chromosome 10 pictogram (left part) with position of PTEN and MGMT genes and enlargement of the 10q25.3-26.1 area between markers D10S562 and D10S214 (right part). Minimally lost regions (3, 7-11) are shown by grey bars (middle), together with sequence tagged site (STS) and expressed sequence tag (EST) maps (right). Scale bar represents 1 megabase (Mb).

Construction of expression plasmids. GPR26 cDNA obtained by RT-PCR was cloned into the expression plasmid pcDNA3.1 and sequenced. Open reading frames for enhanced green fluorescent protein (EGFP) and MYC tags were fused in-frame to the $3^{\prime}$ end of the GPR26 coding sequence devoid of stop codon.

Micro-array analysis of glioma $m R N A$. Total RNA from 12 GBMI, 3 GBMII, and 8 astrocytomas (AS) was amplified and labelled using the Affymetrix 2-cycle amplification protocol as per manufacturer's instructions (Affymetrix). Samples were hybridized to Affymetrix U133v2.0 GeneChips and scanned using an Affymetrix GeneChip scanner as per manufacturer's instructions. Expression values were estimated using the GC-RMA implementation found in the Genedata Refiner 4.1 (Genedata, Basel, Switzerland) package. Datamining and visualization was performed using the Genedata Analyst 4.1 package. All samples were quantile normalized and median scaled to correct for minor variations in their expression distributions.

Search for somatic GPR26 mutations. Coding regions of exons 1, 2 and 3 were sequenced from amplified genomic DNA of cell lines LN18, LN235, LNz308, LN401, LN427, LN428, U87, U343 and primary tumors BS6T, BS13T and $\mathrm{BS} 30$ using the following primers. Exon 1: cetgagcgecggcgc 
A

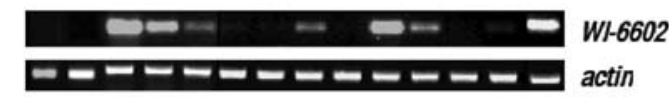

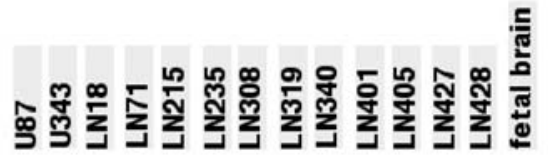

C

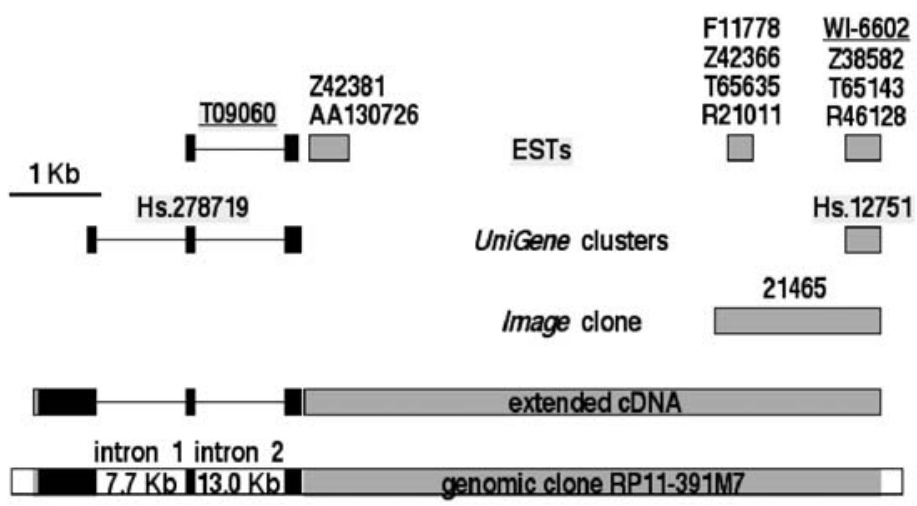

B

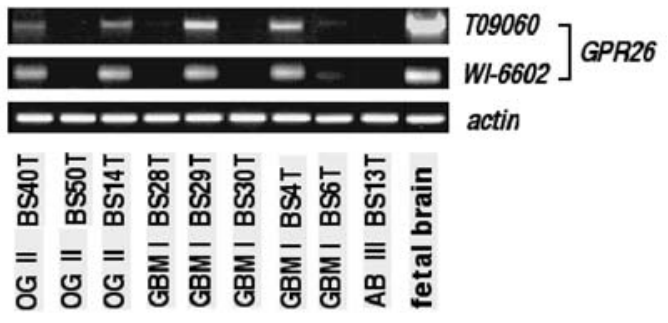

D

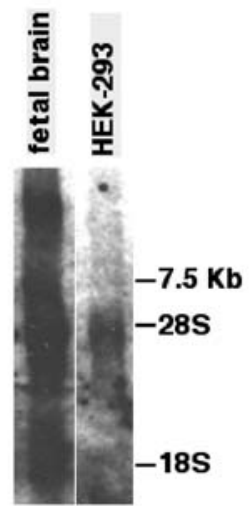

Figure 2. EST WI-6602 is part of the GPR26 transcription unit. (A) WI-6602 (upper panel) and actin (lower panel) cDNA detection in glioma cell lines by PCR and ethidium bromide staining of agarose gel; (B) T09060 (upper panel), WI-6602 (middle panel) and actin (lower panel) cDNA expression in primary gliomas. WHO tumor grades (II-IV) and differentiation of primary gliomas are indicated. AB, astroblastoma; AS astrocytoma; GBMI, primary glioblastoma; GBMII, secondary glioblastoma; (C) Top, expressed sequence tags (ESTs) and UniGene clusters of overlapping ESTs. Middle, 5' extended cDNA. The sequence of phagemid Image clone 21465 was completed and 5 ' extended, resulting in a $6.6 \mathrm{~kb}$ cDNA, with a $100 \%$ continuous homology with the human chromosome 10 genomic clone RP11-391M7 and overlap at its 5'-end with the infant brain-specific UniGene cluster Hs.278719. Confirmation that both Hs.278719 and extended Hs.12751 transcribed are part of the same transcription unit was provided by RT-PCR. Bottom, more 5'-end sequences were identified based on a $92 \%$ sequence homology between the UniGene cluster Hs.278719 and the rat orphan G protein-coupled receptor GPR26 (20). GPR26 coding region is indicated by filled boxes, introns are not to scale; (D) Size of the WI-6602 transcripts. Northern blotting of $15 \mu \mathrm{g}$ total RNAs from fetal brain and from the embryonic kidney cell line HEK293 probed with cDNA clone 21465 containing WI-6602.

ggggcgcg and ctcceactccegccgagatccegcgaggct; exon 2: gccaaggtcaatagcta and gacacacctgccaatcaggc; exon 3: ggctagtggagctcttctccacggtg and gagacgaggtgccatgttagatc.

Restoration of GPR26 expression. Glioma cell lines were plated at low-density and incubated with $1 \mu \mathrm{M}$ 5-aza-2'deoxycytidine (AZA), and/or $1 \mu \mathrm{M}$ trichostatin A (TSA). Optimal conditions were found to be a 48-h treatment with AZA, and TSA added $6 \mathrm{~h}$ before cells were harvested. GPR26 expression was assessed using GPR26 (gccaccaagaagatcagca ccttca and gagacgaggtgccatgttagatc) and $A C T B$ (ggtgtaacgcaa ctaagtcatag and gcatggagtcctgtggcatccacg) primers as internal control on reverse-transcribed RNA. PCR products were separated on agarose gels. GPR26 products were also subjected to Southern blotting.

DNA modification. Tumor genomic DNA was modified with bisulfite following the protocol previously described (23). Primers CGGGTACCTTTtTTtgggagTTatgg and CGGGATC caAcaAAcaAaAcaAcacc were used to directly amplifiy and sequence modified DNA from biopsies, as described in Fig. 4.

Intracellular cAMP measurement. Analysis was performed with the commercially available kit cAMP Direct Biotrak (Amersham, Piscataway, NJ, USA) following manufacturer's instruction. Standard deviations were calculated based on 2 measurements.
Cell proliferation assay. Bromodeoxyuridine (BrdU) was added to the medium $1 \mathrm{~h}$ before cell harvesting at $10 \mu \mathrm{mol} / \mathrm{l}$ concentration. Cells were labeled with an anti-BrdU antibody and DNA staining dye (7-AAD). Fluorescence-activated cell sorting (FACS) analysis was done according to the manufacturer's instructions (Becton Dickinson, Franklin Lakes, NJ).

GenBank accession numbers. The sequence of the human GPR26 mRNA was deposited under the accession number AJ505757 (EMBL), and the deduced peptide sequence under the accession number CAD44281 (Genbank) and Q8NDV2 (SwissProt).

\section{Results}

Identification of the GPR26 transcript. We selected from the region surrounding the 10q25.3-26.1 minimally lost area (3,7,9-11) 26 EST markers and tested them by PCR on genomic and complementary DNAs of glioblastoma cell lines and primary brain tumors of WHO grades II-IV (Fig. 1). No homozygous deletions were detected following amplification of genomic tumor-derived DNA. However, the EST marker WI-6602 showed expression levels comparable to fetal brain in only 3 out of 13 glioma cell lines (23\%), with marked decrease or loss of expression in the other lines (Fig. 2A) and in $2 / 6$ primary malignant gliomas with established allelic 


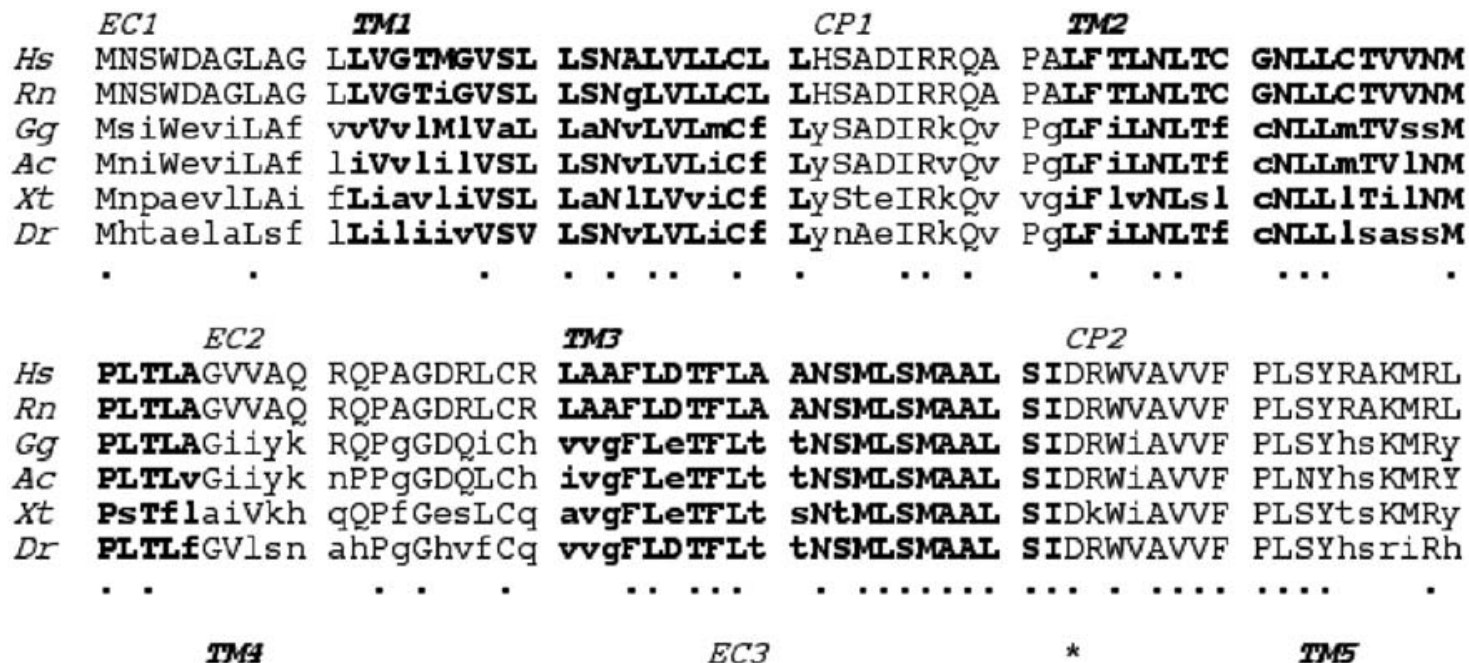

RDAALMVAYT WLHALTTPAA ALALSWLGFH QLYASCTLCS RRPDERLRFA VFTGAFHALS RDAAfMVAYT WLHALTF PAT ALAISWLGFH QLYASCTLCS RRPDERLRFA VFTSAFHAIS RDAALilsYT WLHSVSFPiv AasISWvGFH HLYASCTLYn kRPedRtqFV iFTGvFHtIS kDAALilsYT WLHsvsFPiv AtsLSWvGFH HLYASCTLyn kkledRtqFM IFTGvFHtfS kDAALMmgYs WLHSLTFPlv syffSWLdys smYASCTLha qeeadtrrFm VFTivFHaat RDAvialaYT WvHsLsFsvv AacLswvGyH QqYASCTLCngRavhaktqFA myTlvlHtIt

-

CP3

•.

..... FIISFVVLCC TYLKVLKVAR FHCKRIDVIT MOTLVLLVDL HPSVRERCLE EQKRRRQRAT
FIUSF iVLCf TYLKVLKVAR FHCKRIDVIT MOTLVLLVDi HPSVRERCLE EOKRRRQRAT FILSIiVLCf TYLKVLKVAR FHCKRIDVIT MOTLVLLVDi HPSVRERCLE EQRKRRQRAT FIISIVVLCF TYLKVLKVAR FHCKRIDVIT MOTLVLLVDI HPSVRERCLE EQKRRRQRAT FmIS11i工cf TYLKVLKVAR FHCrRIDIIT MQTLVLLVDL HPSVkqRCLS EQKRRRQRAT FLLVSVVLCv TYLKVLKVAR FHCKRIDVIT MOTLVLLVDi HPSVRqRCLE EQKRRRQRAT $\begin{array}{lllllllllllllllll} & . & \ldots & \ldots & \ldots & \ldots & \ldots & \ldots & \ldots & \ldots & \ldots & \ldots & \ldots & \ldots & \ldots & \ldots & \ldots\end{array}$

TM6 $\downarrow E C 4 \quad$ TM7

HS KKISTF IGTF LVCFAPYVIT RLVELFSTVP IGSHWGVLSK CLAYSKAASD PFVYSILRHO $R n$ KKISTF IGTF LVCFAPYVIT RLVELFSTAP IdSHWGVLSK CLAYSKAASD PFVYSIIRHQ

Gg KKISTF IGTF ilCFAPYVIT RLVELSSTIP InSHWGiiSK CLAYSKVVSD PFVYSILRnQ

AC KKISTFIGTF VICFAPYVIT RLVELSSVAP InAHWGVISK CLAYSKAVSD PFVYSIIRHO

Xt KKISvF IGSF vVCFAPYVvT RLiELlpfVk InIYWGivSK CLAYSKAASD PFVYSILRqQ

DI IKISTF IGTF VVCFSPYVIT RIVELFITEP f NPYWGVLCK SIAYSKAACD PFVYSIIRHQ

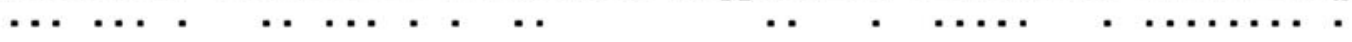

HS YRKSCKEILN RLLHRRSIHS SGLTGDSHSQ NILPVSE]

$R n$ YRISCKEILN RifnRRSIHS VGLTGDSHSQ NILPVSE]

Gg YkKtwKdIiN kiLkRsSInS SaLTsesHnr NILqlnE]

Ac YkKtwKdIiN kvLkRsSInS SaLTseSqSr NILqVnE]

$X t$ YknvllnIvN RvLkRelyps SGynssldte Ndyclhrps]

Dr YRKtCsdIiN RlmkRsSlng Srhqqqngki vrakeigkhg dvegrpkd]

Figure 3. Sequence similarities between vertebrate GPR26 orthologues. Primary sequence alignment of human (Hs) CAD44281; rat (Rn) AAF21012; bird (Gallus gallus, Gg) XP_421809; reptilian (Anolis carolinensis, Ac); amphibian (Xenopus tropicalis, Xt) and bone fish (Danio rerio, Dr) XP_693338. Reptile GPR26 was reconstituted by tBlastn search on translated Anolis carolinensis genome with the human sequence CAD44281. Amino acid one-letter code used. Evolutionary conserved amino acids are uppercase and highlighted with dots; amino acids divergent from the human sequence are lowercase and grey; transmembrane domains (TMs) described by Lee et al (20) are bold; extra-cytoplasmic (EC) and cytoplasmic (CP) domains are plain. Arrows indicate codons S223 and R261 interrupted by exon boundaries. Asterisk indicates codon P163 in which the synonymous single nucleotide polymorphism CCG $\rightarrow$ CCA (rs12263344) was observed.

loss in the 10q25.3-26.1 area (9) (Fig. 2B, middle row). Genomic position of WI-6620 validates the compilation of the minimally areas of loss shown in Fig. 1.

WI-6602 is part of the UniGene cluster Hs.12751 (Fig. 2C, top) expressed in infant brain. 5' extension of this cDNA gave rise to a 7512-bp cDNA that also covered the infant brainexpressed UniGene cluster Hs.278719, sequence of which displays $92 \%$ nucleotide sequence homology with the cDNA encoding the rat orphan $\mathrm{G}$ protein-coupled receptor GPR26 (20). RT-PCR revealed also consistent expression profiles between GPR26 EST markers WI-6602 and T09060 in primary human glial tumors (Fig. 2C). Definition of a single transcription unit that covers both Unigene clusters was in agreement with the $7.5 \mathrm{~Kb}$-long transcript size detected by 
Table I. Status of human gliomas at SNP rs12263344.

\begin{tabular}{|c|c|c|}
\hline Glioma & & SNP status \\
\hline \multicolumn{3}{|l|}{ GBM cell lines } \\
\hline U87 & & P163: CCG \\
\hline U343 & & P163: CCA \\
\hline LN18 & & P163: CCG \\
\hline LN235 & & P163: CCA \\
\hline LN308 & & P163: CCG \\
\hline LN401 & & P163: CCA \\
\hline LN427 & & P163: CCA \\
\hline LN428 & & P163: CCG \\
\hline Primary tumors & Tumor type/grade & SNP status \\
\hline BS13T & AB III & P163: CCG \\
\hline BS6T & GBM I & P163: CCA \\
\hline BS30T & GBM I & P163: CCA \\
\hline
\end{tabular}

GBM I, primary gliobastoma; AB, astroblastoma WHO grade III.

Northern blot analysis of fetal brain RNA probed with the Image cDNA clone 21465 (Fig. 2D).

GPR26 contains two introns that split the coding sequence of 1014 nucleotides into three parts of 668, 114 and 232 nucleotides respectively, at codons 223 and 321 (Fig. 3, arrows). The encoded GPR26 protein consists of 337 amino acids $96 \%$ identical with rat gpr26. Comparison with other verebrate orthologues shows the highest sequence conservation at the third cytoplasmic domain (Fig. 3). We sequenced the GPR26 coding region from eight glioblastoma cell lines and three primary brain tumors with allelic loss in this area $(3,9)$. No mutations were detected, except for the synonymous single nucleotide polymorphism rs 12263344 at codon encoding proline 163 (CCG $\rightarrow \mathrm{CCA}$ ) detected in $6 / 11$ of tumors as well as in corresponding germline lymphocyte DNA (Fig. 3, star; Table I). Given that the upstream region of the GPR26 gene contains a dense $\mathrm{CpG}$ island (Fig. 4, top), (24) that the $10 \mathrm{q} 25.3-\mathrm{q} 26.1$ gene $M G M T$ is frequently subject to frequent silencing by hypermethylation in gliomas (17) and that epigenetic silencing in cancer can cover chromosomal regions of several megabases, we hypothesized that GPR26 was inactivated by epigenetic silencing.

Re-expression of GPR26 by 5-aza-deoxycytidine and/or trichostatin A. We analyzed RNA expression of GPR26 and $M G M T$ genes, both located within 6 megabases of the telomere of chromosome $10 \mathrm{q}$ on 23 primary glioma microarrays. Definition of a cut-off of 70 GC-RMA expression values for both transcripts showed highly significant $(\mathrm{p}=0.0001)$ co-expression of GPR26 and MGMT, suggesting a common mechanism of down-regulation (Fig. 4). On the other hand, assessment of the methylation status of the $5^{\prime} \mathrm{CpG}$ island (23) of GPR26 in 14 primary gliomas revealed a cluster of

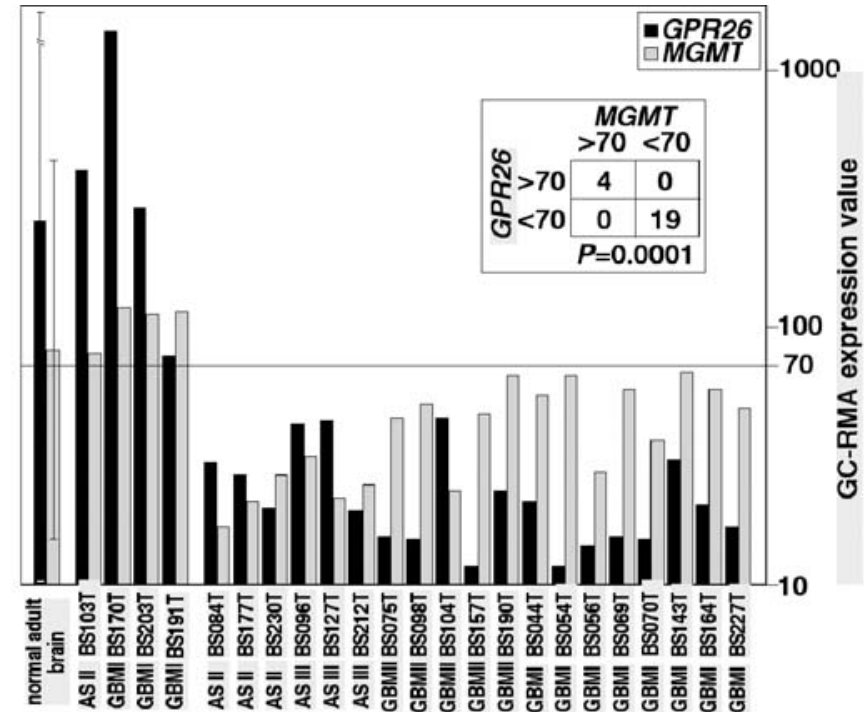

Figure 4. Co-expression of GPR26 and MGMT in primary gliomas. Microarray expression analysis of GPR26 (black) and MGMT (grey) on microarrays of 23 primary gliomas. GC-RMA expression values for GPR26 (Affymetrix probeset ID 244617_at) and $M G M T$ (Affymetrix probeset ID 204880_at) transcripts are shown on the y-axis. Significant co-expression ( $p=0.0001$, Fisher's exact test) is shown by using a cut-off of 70 (thicker line) between low and high expression levels for both transcripts.

methylated $\mathrm{CpG}$ within 60 nucleotides surrounding the GPR26 translation initiation codon (Fig. 5A). Moreover, comparison of the methylation status of this area with GPR26 expression profiles in 9 glioma cells and biopsies indicates a correlation between complete methylation of the $\mathrm{CpG}$ doublet located 12 bp downstream of the ATG codon and little or no GPR26 expression. In contrast, limited or no methylation is needed for GPR26 expression as shown for LN18 and LN71 (Figs. 2A and 5B), but not sufficient, as seen in LN308. Addition of the methylation inhibitor 5-aza-deoxycytidine (AZA) into culture medium restored GPR26 expression in U87 cells, while the histone deacetylase inhibitor trichostatin A (TSA) was required in U343 cells (Fig. 5C), suggesting that histone deacetylation is an additional contributor of GPR26 epigenetic silencing.

Re-expression of GPR26 increases cAMP levels. G proteincoupled receptor signaling is mediated via cAMP increase. Initial transfection of the GPR26-negative HEK293 cells, routinely used for cAMP studies (25), with the human GPR26 cDNA resulted in a 7-fold increase of intracellular cAMP levels (Fig. 6A). In U87 cells, that do not express GPR26 mRNA either, intracellular cAMP levels were elevated by a factor of 2 following transfection with a GPR26 expression vector. A previous observation suggested a link between high intracellular cAMP levels and lower proliferative activity in HEK293 cells (20). However, comparison of HEK293 stably transfected to express GPR26 with control HEK293 cells did not show evidence for changes in the distribution of cell cycle phases G0/G1, G2/M and S (Fig. 6B). Since it has been established in rat C6 glioma cells that high intracellular cAMP levels are associated with astrocytic differentiation (26) our data support that GPR26/cAMP signaling may rather play a role in differentiation of human glioma. 

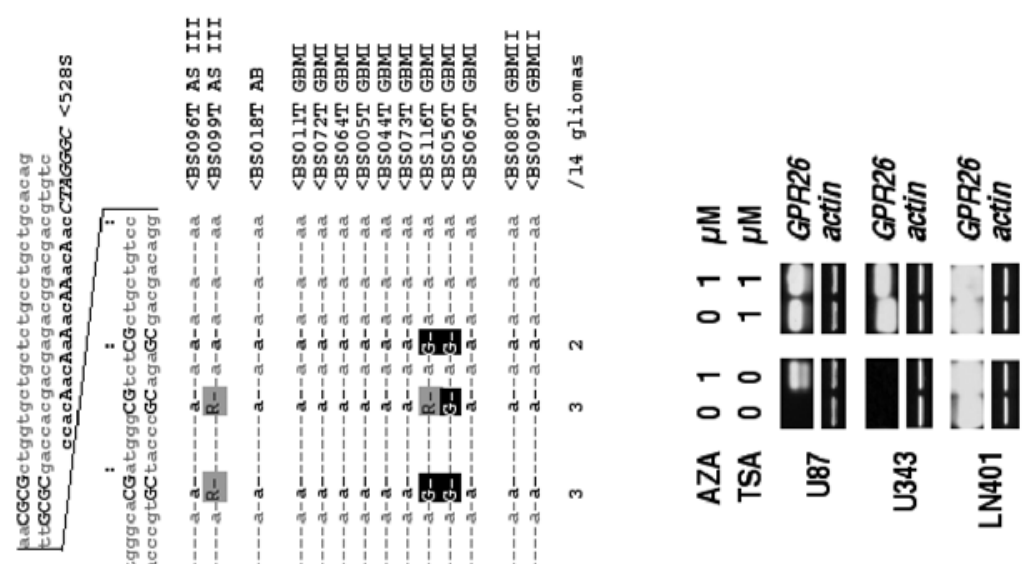

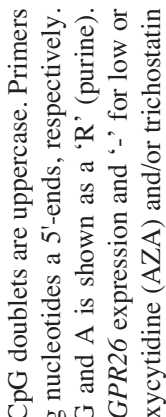

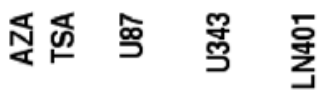

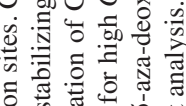

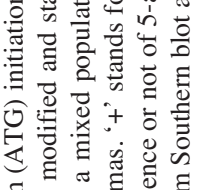

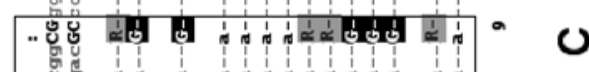

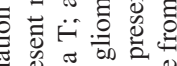

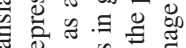

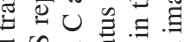

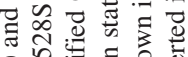

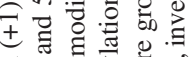

舟

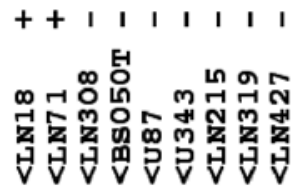

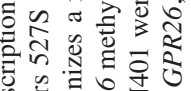

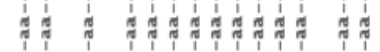

。

象

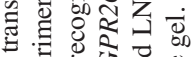

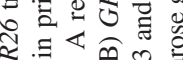

Sิ

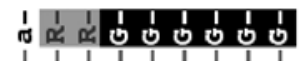

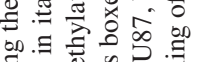

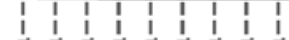

罂:

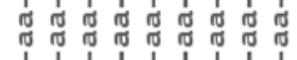

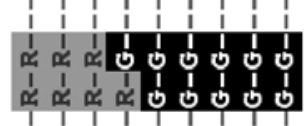

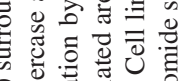

要

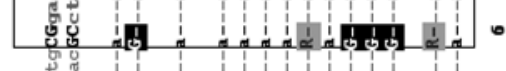

o

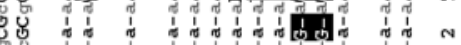

药 细

1.

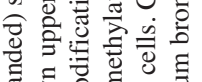

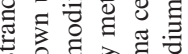

की

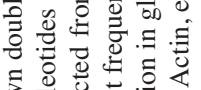

11111

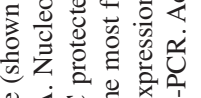

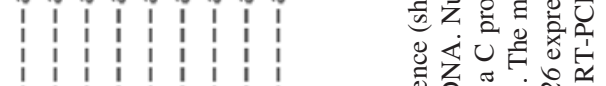

F苾

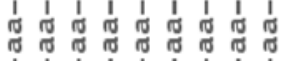

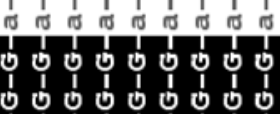

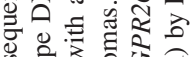

\%

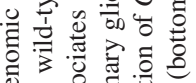

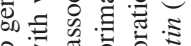

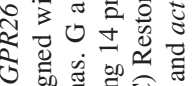

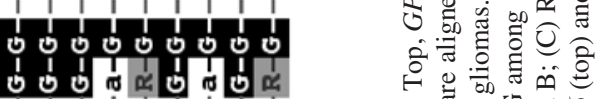

su

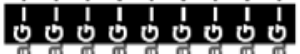

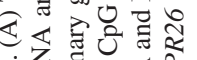

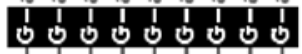

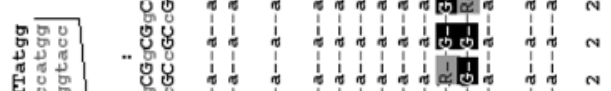

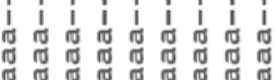

为

100

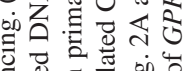

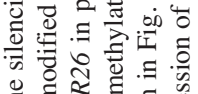

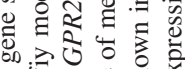

象记

行

응 원

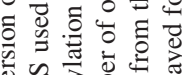

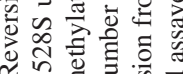

$\approx$ in

的羟

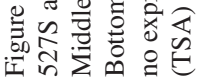


A

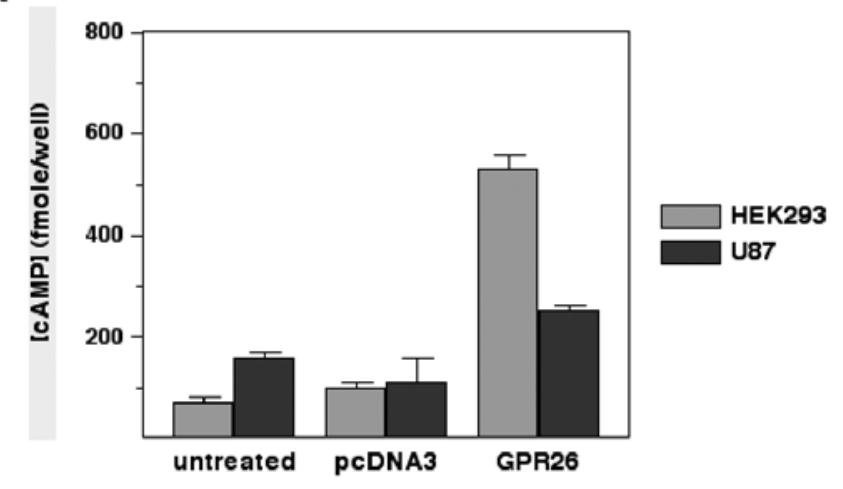

B

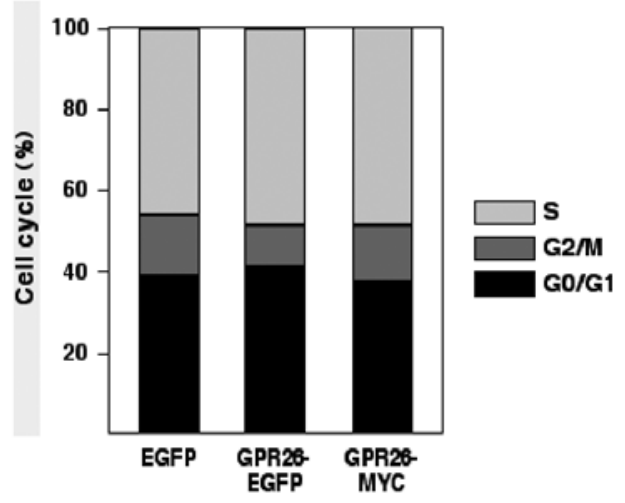

Figure 6. (A) Induction of intracellular cAMP upon GPR26 transfection. cAMP levels in HEK293 and in U87 are shown in light grey and dark grey, respectively; (B) Cell cycle analysis of HEK293 expressing GPR26. Phase distribution of HEK293 cells transfected with a plasmid expressing control enhanced green fluorescent protein (EGFP, left); a plasmid expressing GPR26 tagged by 3' fusion with EGFP (GPR26-EGFP, middle) or a plasmid expressing GPR26 tagged by 3' fusion with MYC (GPR26-MYC, right). Data based on two independent experiments.

\section{Discussion}

Earlier studies delimited the Deleted in Malignant Brain Tumor 1 (DMBT1) locus (10) and the corresponding gene between markers D10S209 and D10S587. However, DMBT1 has been reported not to be specifically targeted in primary gliomas (14), and corresponding transcripts were mainly isolated from tissue of the gastro-intestinal tract and from lung, but, surprisingly not from brain tissue (12). This is also consistent with the two independent studies $(3,9,11)$ that genetically excluded $D M B T 1$ as a suppressor gene. More telomeric, $M G M T$ has been identified as a main target gene for DNA methylation-based inactivation with high prevalence in low-grade astrocytomas $(18,19)$. Since MGMT prevents carcinogenesis by alkylating agents (15) and its gene frequently inactivated in gliomas, MGMT is a critical 10q25.3-26.1 tumor suppressor operative in brain.

GPR26 is located within the smallest interval consistent with all the somatic deletion studies reported on distal 10q in gliomas (Fig. 1). On the other hand, 10q25.3-26.1 epigenetic silencing appears to potentially inactivate a large array of genes in brain tumors. Moreover, EST markers contained within the GPR26 transcript were exclusively isolated from the infant brain cDNA library (UniGene Library No. 37), and are highly expressed in human adult brain as well as in rat adult brain (20). Thus, GPR26 expression profile in normal brain and frequent absence of expression in gliomas would also be compatible with a tumor suppression function specific of the glial lineage. In the particular case of GPR26, hemizygous loss in conjunction with epigenetic silencing of the 10 q25.3-26.1 area of the remaining copy would be needed for gene inactivation in brain tumors.

Gene silencing by aberrant promoter methylation has been extensively reported in various tumor types, including glioma (27-31). It has recently been shown that $\mathrm{CpG}$ methylation in colorectal cancer can span several megabases, thereby silencing expression of genes located in a same region (24). On the other hand, TSA inhibits histone deacetylase, a process required for chromatin decondensation and initiation of gene transcription (32). Interestingly, histone deacetylase is a promising drug target for cancer treatment (33). In fact, both epigenetic mechanisms have been linked to the transcriptional repressor MeCP2 (34). It is not clear whether TSA alone can restore expression of methylated genes, or whether synergy with 5-aza-deoxycytidine is required (35). Our data show that, for example in glioblastoma cell lines U87 and U343, TSA alone can be sufficient to induce re-expression of GPR26.

Whether GBM occur de novo or derive from a lower grade astrocytoma has suggested the clinical distinction between primary and secondary GBM, respectively. This classification has been further supported by distinct prevalences of altered glioma pathways (21). Based on the prevalence of PTEN (10q23) loss in primary GBM, GPR26 (10q25-26) expression has recently been proposed as a recognition marker between primary and secondary GBM, based on the observation that expression of this gene is reduced with increasing age (36). Among our samples, 25/32 (78\%) of primary GBM and 2/3 (67\%) of secondary GBM had 10q loss (9). We also observed parallel expression between GPR26 and MGMT ( $\mathrm{p}=0.0001)$ (Fig. 4), while MGMT (10q26) low expression and TP53 mutation are frequently associated in secondary GBM (21). However, we found GPR26 expression to be reduced or lost in all glioblastoma samples whether they were primary and secondary GBMs $(9,21)$. Likewise, 10q deletion and epigenetic silencing was found in both primary and secondary GBM (21). We therefore believe that definition of the GPR26 status in a larger number of GBM may help to clarify this issue and possibly to define a molecular subset of GBM.

Upon GRP26 transfection, HEK293 cells showed a marked increase of intracellular cAMP levels while elevation of cAMP levels was 2-fold in U87 glioblastoma cells. The attenuated response in U87 cells may be due to the low transfection efficiency of glioma cells, or the absence of putative GPR26 ligand. Although the cognate ligand for the orphan G-proteincoupled GPR26 remains to be identified, the fact that GPR26 has close homology to the serotonin receptor 5-HT5A identifies GPR26 as a member of the amine-like receptor subfamily of $\mathrm{G}$ protein-coupled receptors. In addition, full activation of this signaling pathway may need additional components that can also be lacking in glioblastoma cells. Indeed, as 
described for other $\mathrm{G}$ protein-coupled receptors that are parts of multi-chain receptor complexes, GPR26 forms a heterooligomer with the serotonin receptor 5-HT1A $(37,38)$.

Possible roles of GPR26/cAMP signaling in tumorigenesis have been suggested. A single study has showed that expression of rat gpr 26 reduced the growth rate and induces morphological changes in HEK293 cells (20). However, in our hands, GPR26 expression in HEK293 cells did not alter distribution of cell cycle phases. Other observations established that high intracellular cAMP levels are associated with astrocytic differentiation in rat cortical precursor cells (26). In addition, defects in cAMP pathway were shown to potentially initiate carcinogenesis in cells of the central nervous system (39). Our data show that GPR26 together with MGMT belong to a region on $10 \mathrm{q} 25.3-26.1$ that is frequently inactivated by hemizygous deletion and epigenetic silencing of the residual alleles. This region may therefore represent an important epigenetic pathway in brain tumorigenesis.

\section{Acknowledgements}

This work was supported by the Swiss National Foundation for Scientific Research (SNF 30053746.98), OncoSuisse (OCS-01613-12-2004), the Krebsliga Beider Basel (No. 7 2004) and the Bernardi Trust Foundations. The Friedrich Miescher Institute is supported by the Novartis Science Foundation. We would like to thank Herbert Angliker and Edward Oakeley for their help with running the microarrays and analyzing the raw data, respectively.

\section{References}

1. Knobbe $\mathrm{CB}$, Merlo A and Reifenberger G: Pten signaling in gliomas. Neurooncology 4: 196-211, 2002.

2. Li J, Yen C, Liaw D, Podsypanina K, Bose S, Wang SI, Puc J, Miliaresis C, Rodgers L, McCombie R, Bigner SH, Giovanella BC, Ittmann M, Tycko B, Hibshoosh H, Wigler MH and Parsons R: Pten, a putative protein tyrosine phosphatase gene mutated in human brain, breast, and prostate cancer. Science 275: 1943-1947, 1997.

3. Maier D, Zhang Z, Taylor E, Hamou MF, Gratzl O, van Meir EG, Scott RJ and Merlo A: Somatic deletion mapping on chromosome 10 and sequence analysis of pten/mmacl point to the 10q25-26 region as the primary target in low-grade and highgrade gliomas. Oncogene 16: 3331-3335, 1998

4. Sano T, Lin H, Chen X, Langford LA, Koul D, Bondy ML, Hess KR, Myers JN, Hong YK, Yung WK and Steck PA: Differential expression of $\mathrm{mmac} / \mathrm{pten}$ in glioblastoma multiforme: relationship to localization and prognosis. Cancer Res 59: $1820-1824,1999$.

5. Steck PA, Pershouse MA, Jasser SA, Yung WK, Lin H, Ligon AH, Langford LA, Baumgard ML, Hattier T, Davis T, Frye C, Hu R, Swedlund B, Teng DH and Tavtigian SV: Identification of a candidate tumour suppressor gene, mmac1, at chromosome 10q23.3 that is mutated in multiple advanced cancers. Nat Genet 15: 356-362, 1997.

6. Tamura M, Gu J, Matsumoto K, Aota S, Parsons R and Yamada KM: Inhibition of cell migration, spreading, and focal adhesions by tumor suppressor pten. Science 280: 1614-1617, 1998

7. Albarosa R, Colombo BM, Roz L, Magnani I, Pollo B, Cirenei N, Giani C, Conti AM, DiDonato S and Finocchiaro G: Deletion mapping of gliomas suggest the presence of two small regions for candidate tumor-suppressor genes in a $17-\mathrm{cm}$ interval on chromosome 10q. Am J Hum Genet 58: 1260-1267, 1996.

8. Fults D and Pedone C: Deletion mapping of the long arm of chromosome 10 in glioblastoma multiforme. Genes Chromosomes Cancer 7: 173-177, 1993.
9. Maier D, Comparone D, Taylor E, Zhang Z, Gratzl O, van Meir EG, Scott RJ and Merlo A: New deletion in low-grade oligodendroglioma at the glioblastoma suppressor locus on chromosome 10q25-26. Oncogene 15: 997-1000, 1997.

10. Moschonas NK, Spurr NK and Mao J: Report of the first international workshop on human chromosome 10 mapping 1995. Cytogenet Cell Genet 72: 100-112, 1996.

11. Rasheed BK, McLendon RE, Friedman HS, Friedman AH, Fuchs HE, Bigner DD and Bigner SH: Chromosome 10 deletion mapping in human gliomas: a common deletion region in 10q25. Oncogene 10: 2243-2246, 1995.

12. Mollenhauer J, Wiemann S, Scheurlen W, Korn B, Hayashi Y, Wilgenbus KK, von Deimling A and Poustka A: Dmbt1, a new member of the SRCR superfamily, on chromosome 10q25.326.1 is deleted in malignant brain tumours. Nat Genet 17: 32-39, 1997.

13. Wu W, Kemp BL, Proctor ML, Gazdar AF, Minna JD, Hong WK and Mao L: Expression of dmbt1, a candidate tumor suppressor gene, is frequently lost in lung cancer. Cancer Res 59: 1846-1851, 1999.

14. Sasaki H, Betensky RA, Cairncross JG and Louis DN: Dmbt1 polymorphisms: relationship to malignant glioma tumorigenesis. Cancer Res 62: 1790-1796, 2002.

15. Goth R and Rajewsky MF: Persistence of $\mathrm{O}^{6}$-ethylguanine in rat-brain DNA: correlation with nervous system-specific carcinogenesis by ethylnitrosourea. Proc Natl Acad Sci USA 71: 639-643, 1974 .

16. Inoue R, Isono M, Abe M, Abe T and Kobayashi H: A genotype of the polymorphic DNA repair gene MGMT is associated with de novo glioblastoma. Neurol Res 25: 875-879, 2003.

17. Costello JF, Futscher BW, Tano K, Graunke DM and Pieper RO: Graded methylation in the promoter and body of the O6methylguanine DNA methyltransferase (MGMT) gene correlates with mgmt expression in human glioma cells. J Biol Chem 269: 17228-17237, 1994.

18. Hegi ME, Diserens AC, Gorlia T, Hamou MF, De Tribolet N, Weller M, Kros JM, Hainfellner JA, Mason W, Mariani L, Bromberg JE, Hau P, Mirimanoff RO, Cairncross JG, Janzer RC and Stupp R: Mgmt gene silencing and benefit from temozolomide in glioblastoma. N Engl J Med 352: 997-1003, 2005.

19. Watts GS, Pieper RO, Costello JF, Peng YM, Dalton WS and Futscher BW: Methylation of discrete regions of the $\mathrm{O}^{6}$-methylguanine DNA methyltransferase (MGMT) CPG island is associated with heterochromatinization of the MGMT transcription start site and silencing of the gene. Mol Cell Biol 17: 5612-5619, 1997 .

20. Lee DK, Lynch KR, Nguyen T, Im DS, Cheng R, Saldivia VR, Liu Y, Liu IS, Heng HH, Seeman P, George SR, O'Dowd BF and Marchese A: Cloning and characterization of additional members of the $g$ protein-coupled receptor family. Biochim Biophys Acta 1490: 311-323, 2000.

21. Ohgaki $\mathrm{H}$ and Kleihues P: Genetic pathways to primary and secondary glioblastoma. Am J Pathol 170: 1445-1453, 2007.

22. Ishii N, Maier D, Merlo A, Tada M, Sawamura Y, Diserens AC and Van Meir EG: Frequent co-alterations of tp53, p16/cdkn2a, p14arf, pten tumor suppressor genes in human glioma cell lines. Brain Pathol 9: 469-479, 1999.

23. Herman JG, Graff JR, Myohanen S, Nelkin BD and Baylin SB: Methylation-specific PCR: a novel PCR assay for methylation status of CPG islands. Proc Natl Acad Sci USA 93: 9821-9826, 1996.

24. Frigola J, Song J, Stirzaker C, Hinshelwood RA, Peinado MA and Clark SJ: Epigenetic remodeling in colorectal cancer results in coordinate gene suppression across an entire chromosome band. Nat Genet 38: 540-549, 2006.

25. Bresnick JN, Skynner HA, Chapman KL, Jack AD, Zamiara E, Negulescu P, Beaumont K, Patel S and McAllister G: Identification of signal transduction pathways used by orphan $\mathrm{g}$ protein-coupled receptors. Assay Drug Dev Technol 1: 239-249, 2003.

26. Vallejo I and Vallejo M: Pituitary adenylate cyclase-activating polypeptide induces astrocyte differentiation of precursor cells from developing cerebral cortex. Mol Cell Neurosci 21: 671-683, 2002.

27. Graff JR, Herman JG, Lapidus RG, Chopra H, Xu R, Jarrard DF, Isaacs WB, Pitha PM, Davidson NE and Baylin SB: E-cadherin expression is silenced by DNA hypermethylation in human breast and prostate carcinomas. Cancer Res 55: 5195-5199, 1995. 
28. Herman JG, Merlo A, Mao L, Lapidus RG, Issa JP, Davidson NE, Sidransky D and Baylin SB: Inactivation of the cdkn2/p16/mts1 gene is frequently associated with aberrant DNA methylation in all common human cancers. Cancer Res 55: 4525-4530, 1995

29. Herman JG, Umar A, Polyak K, Graff JR, Ahuja N, Issa JP, Markowitz S, Willson JK, Hamilton SR, Kinzler KW, Kane MF, Kolodner RD, Vogelstein B, Kunkel TA and Baylin SB: Incidence and functional consequences of hmlh 1 promoter hypermethylation in colorectal carcinoma. Proc Natl Acad Sci USA 95: 6870-6875, 1998.

30. Merlo A, Herman JG, Mao L, Lee DJ, Gabrielson E, Burger PC Baylin SB and Sidransky D: 5' CPG island methylation is associated with transcriptional silencing of the tumour suppressor $\mathrm{p} 16 / \mathrm{cdkn} 2 / \mathrm{mts} 1$ in human cancers. Nat Med 1: 686692, 1995.

31. Yoshikawa H, Matsubara K, Qian GS, Jackson P, Groopman JD, Manning JE, Harris CC and Herman JG: Socs-1, a negative regulator of the jak/stat pathway, is silenced by methylation in human hepatocellular carcinoma and shows growth-suppression activity. Nat Genet 28: 29-35, 2001.

32. Jones PA and Baylin SB: The fundamental role of epigenetic events in cancer. Nat Rev Genet 3: 415-428, 2002.

33. Yoshida M, Furumai R, Nishiyama M, Komatsu Y, Nishino N and Horinouchi S: Histone deacetylase as a new target for cancer chemotherapy. Cancer Chemother Pharmacol 48 (Suppl. 1): S20-S26, 2001.
34. Nan X, Ng HH, Johnson CA, Laherty CD, Turner BM, Eisenman RN and Bird A: Transcriptional repression by the methyl-CPG-binding protein MECP2 involves a histone deacetylase complex. Nature 393: 386-389, 1998.

35. Cameron EE, Bachman KE, Myohanen S, Herman JG and Baylin SB: Synergy of demethylation and histone deacetylase inhibition in the re-expression of genes silenced in cancer. Nat Genet 21: 103-107, 1999.

36. Carter AN, Cole CL, Playle AG, Ramsay EJ and Shervington AA Gpr26: A marker for primary glioblastoma? Mol Cell Probes 22: 133-137, 2008.

37. Park PS, Filipek S, Wells JW and Palczewski K: Oligomerization of g protein-coupled receptors: past, present, and future. Biochemistry 43: 15643-15656, 2004.

38. Salim K, Fenton T, Bacha J, Urien-Rodriguez H, Bonnert T, Skynner HA, Watts E, Kerby J, Heald A, Beer M, McAllister G and Guest PC: Oligomerization of g-protein-coupled receptors shown by selective co-immunoprecipitation. J Biol Chem 277: 15482-15485, 2002.

39. Prasad KN, Cole WC, Yan XD, Nahreini P, Kumar B, Hanson A and Prasad JE: Defects in camp-pathway may initiate carcinogenesis in dividing nerve cells: A review. Apoptosis 8: 579-586, 2003. 